\title{
Fluid Dynamics and Mass Transfer in a Perfusion Bioreactor with a Porous Wall
}

\author{
H. T. Low, H. X. Bai, P. Yu, Y. Zeng, and S. H. Winoto
}

\begin{abstract}
A numerical study is carried out on the fluid dynamics and mass transfer in a microchannel perfusion bioreactor. The bioreactor channel has a porous wall for the co-culture of two types of cells which are distributed randomly and uniformly. A group of dimensionless parameters is proposed, which can be applied to correlate the numerical data and characterize the mass transfer in the bioreactor. The normalized numerical data, for the concentration at the porous-fluid interface and concentration difference between the interface and the base, show satisfactory correlation when presented as a function of the effective distance parameter.
\end{abstract}

Index Terms-Transport phenomena in porous media, numerical methods in fluid flow, mass transfer.

\section{INTRODUCTION}

In a type of perfusion bioreactor design, a porous layer partially occupies the channel [1], [2]. It was found [3] that the shear stress in this bioreactor was significantly lower than others, which made their bioreactor suitable for cell cultures sensitive to shear stress. A model was developed [4], [5] for mass transport in the perfusion channel partially filled with a porous layer, in which the flow convection in the porous layer has been neglected. The model was improved [3] by applying Brinkman's model in the porous layer. By assuming the continuities of velocity and shear stress at the porous-fluid interface, the improved model was numerically solved by the Lattice-Boltzmann method. More recently, a two-domain approach [6] was applied to simulate flow and mass transport in this type of bioreactor. The Navier-Stokes equations and the generalized Darcy-Brinkman-Forchheimer extended equations were applied to govern the flows in the fluid and porous regions, respectively.

The present paper numerically investigates flow and mass transfer in a perfusion channel partially filled with a porous layer, which is suitable for co-culture applications. One of the main objectives of the present study is to propose a group of combined-parameters that are useful for the design of this type of bioreactor.

Manuscript received November 29, 2013; revised February 28, 2014

H. T. Low and S. H. Winoto are with the Department of Mechanical Engineering, National University of Singapore, Singapore 117575 (e-mail: mpelowht@nus.edu.sg, mpewinot@nus.edu.sg).

H. X. Bai was with the National University of Singapore, Singapore 117575.

$\mathrm{P} . \mathrm{Yu}$ is with the Data Storage Institute (DSI), A*STAR, Singapore 117608 (e-mail: Yu_Peng@dsi.a-star.edu.sg).

Y. Zeng is with the Nanyang Technological University, Singapore 639798 (e-mail: ZENGYAN@ntu.edu.sg).

\section{NUMERICAL MODEL}

The bioreactor modelled in the present study consists of a channel partially filled with a porous medium as shown in Fig. 1. The width of the channel, being much larger than the depth, has negligible effect [7]. Thus the numerical model considered here is simplified into a two-dimensional one like that of a previous study [4]. The length of the channel is $L$. The depths of the porous and homogenous fluid regions are $h$ and $H$, respectively. In the present configuration, the length scale is much larger than the depth scale so that the inlet effect can be neglected.

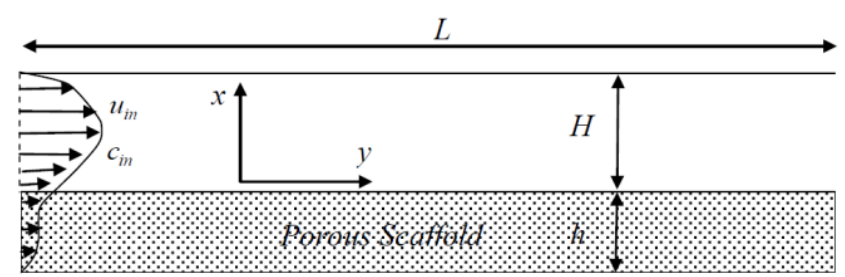

Fig. 1. Schematic of the bioreactor model (not to scale).

The culture medium flows through the channel along the $x$ direction. The absorption and release cells adherent to the porous medium scaffold consume and secrete the substrate respectively, forming the reactions in the porous scaffold. The incoming flow with substrate concentration $c_{\text {in }}$ is steady, laminar and incompressible. The fully developed velocity profile is imposed at the inlet.

The porous medium is assumed to consist of the scaffold structure and the attached cells, with volume fractions $\varepsilon_{s}$ and $\varepsilon_{c}$ occupied by the scaffold and cells respectively. The porosity of the porous medium $\varepsilon$, which is the void fraction, can be calculated as $\varepsilon=1-\varepsilon_{s}-\varepsilon_{c}$. The cells are assumed to be uniformly distributed in the scaffold. The porous medium is considered to be rigid, homogeneous and isotropic, and saturated with the culture medium. The dimensions of the cells are more than one order smaller than the channel gap and are not considered. Typical cell parameters are given in Tables I and II.

TABLE I: PARAMETERS FOR OXYGEN TRANSPORT AND CONSUMPTION IN

\begin{tabular}{|l|l|c|}
\hline Parameters & Values & \multirow{2}{*}{ References } \\
\hline$D$ & $3.29 \times 10^{-9} \mathrm{~m}^{2} / \mathrm{s}$ & \\
\hline$D_{\text {eff }}$ & $1.59 \times 10^{-9} \mathrm{~m}^{2} / \mathrm{s}$ & \multirow{2}{*}{} \\
\hline$c_{i n}$ & $2.2 \times 10^{-7} \mathrm{~mol} / \mathrm{ml}$ & \\
\hline$V_{m a}$ & $1.25 \times 10^{-17} \mathrm{~mol} / \mathrm{cell} / \mathrm{s}$ & \\
\hline$k_{m}$ & $1.1 \times 10^{-8} \mathrm{~mol} / \mathrm{ml}$ & \\
\hline$\gamma_{a}$ & $5.4 \times 10^{5} \sim 3.6 \times 10^{7}$ cells $/ \mathrm{ml}$ & \\
\hline
\end{tabular}

For the cell reactions, the consumption reaction is assumed to follow the Michaelis-Menten model [13] and the release reaction is assumed to be constant [14]. The governing 
equations for the flow and mass transport [15]-[18] can be written as given below.

TABLE II: PARAMETERS FOR GROWTH FACTOR IN CO-CULTURE

\begin{tabular}{|c|l|c|}
\hline Parameters & \multicolumn{1}{|c|}{ Values } & References \\
\hline$D$ & $4.14 \times 10^{-9} \mathrm{~m}^{2} / \mathrm{s}$ & {$[9]$} \\
\hline$D_{e f f}$ & $2.02 \times 10^{-9} \mathrm{~m}^{2} / \mathrm{s}$ & \multirow{2}{*}{} \\
\hline$c_{i n}$ & $3.4 \times 10^{-8} \mathrm{~mol} / \mathrm{ml}$ & {$[10]$} \\
\hline$V_{m a}$ & $2.2 \times 10^{-25} \mathrm{~mol} / \mathrm{cell} / \mathrm{s}$ & \\
\hline$V_{m r}$ & $3.22 \times 10^{-26} \mathrm{~mol} / \mathrm{cell} / \mathrm{s}$ & Estimated \\
\hline$k_{m}$ & $2.3 \times 10^{-9} \mathrm{~mol} / \mathrm{ml}$ & from [11, 12] \\
\hline$\gamma_{a}$ & $1.0 \times 10^{6} \mathrm{cell} / \mathrm{ml}$ & \multicolumn{2}{c}{} \\
\hline$\gamma_{r}$ & $2.5 .0 \times 10^{6} \mathrm{cell} / \mathrm{s} / \mathrm{ml}$ &
\end{tabular}

For homogenous fluid region:

$$
\begin{gathered}
\nabla \cdot \vec{u}=0 \\
\vec{u} \cdot \nabla \vec{u}=-\frac{1}{\rho} \nabla p+\nu \nabla^{2} \vec{u} \\
\vec{u} \cdot \nabla c=D \nabla^{2} c
\end{gathered}
$$

where $\vec{u}$ is the velocity vector; $p$ is the pressure; $\rho$ and $v$ are the density and fluid viscosity of the culture medium, respectively; and $c$ and $D$ are the concentration and diffusivity of the substrate respectively.

For porous scaffold region:

$$
\begin{gathered}
\nabla \cdot \vec{u}=0 \\
\frac{\vec{u}}{\varepsilon} \cdot \nabla \vec{u}=-\frac{1}{\rho} \nabla(\varepsilon p)+v \nabla^{2} \vec{u}-\frac{\varepsilon v}{K} \vec{u}-\frac{\varepsilon C_{F}|\vec{u}|}{\sqrt{K}} \vec{u} \\
\vec{u} \cdot \nabla c=D_{e f f} \nabla^{2} c-\gamma_{a} V_{m a}\left(\frac{c}{c+k_{m}}-a\right)
\end{gathered}
$$

where $\vec{u}$ is the local average velocity vector (Darcy velocity); $p$ is the local average pressure; $\rho$ and $v$ are the density and the fluid viscosity of the culture medium, respectively; $\varepsilon$ is the porosity; $K$ is the permeability; $C_{F}=1.75 / \sqrt{150 \varepsilon^{3}}$ is the Forchheimer coefficient; $c$ and $D_{\text {eff }}$ are the equilibrium weighted average concentration and the effective diffusivity of the substrate in the porous medium region, respectively; $\gamma_{a}$ is the volume density of the absorb cells; $V_{m a}$ is the maximal substrate uptake rate (SUR); $k_{m}$ is Michaelis-Menten constant or substrate concentration at which the SUR is half-maximal; and $a$ is the ratio of release rate over absorb rate, which is defined as:

$$
a=\frac{V_{m r} \gamma_{r}}{V_{m a} \gamma_{a}}
$$

where $\gamma_{r}$ the volume density of the secretion cell, $V_{m r}$ is the maximal substrate release rate (SRR) for secretion cell. In the present study, we only consider the condition $\frac{c}{c+k_{m}}-a>0$, which indicates that the cell absorption rate is larger than the release rate, and the concentration in the porous scaffold decreases along the flow direction.

For the concentration boundary conditions, zero mass flux condition is imposed on the upper and bottom solid-walls. Uniform concentration of $c_{i n}$ is set at the inlet. As for the outlet, the condition $\mathrm{d} c / \mathrm{d} x=0$ is not satisfied because of the above condition $\frac{c}{c+k_{m}}-a>0$, which indicates that the cell absorption rate is larger than the release rate. Thus, $\partial c^{2} / \partial x^{2}=0$ is imposed at the outlet, which assumes that the concentration drops at a constant rate near the outlet boundary region. At the interface between the homogeneous fluid region and porous media scaffold, continuities of mass and mass flux [19] are imposed.

For the velocity boundary conditions, the non-slip condition is imposed on the solid upper and bottom walls. As we are considering the steady laminar channel flow, to reduce computation cost, a fully-developed flow condition is imposed for the channel inlet. At the interface between the homogeneous fluid region and porous media scaffold, besides the continuity of velocity, the shear stress jump condition [20] and the continuity of normal stress condition are imposed:

$$
\begin{gathered}
\left.\frac{1}{\varepsilon} \frac{\partial u_{t}}{\partial n}\right|_{\text {porous }}-\left.\frac{\partial u_{t}}{\partial n}\right|_{\text {fluid }}=\left.\frac{\beta}{\sqrt{K}} u_{t}\right|_{\text {interface }}+\frac{\beta_{1} u_{t}^{2}}{v} \\
\left.\frac{v}{\varepsilon} \frac{\partial u_{n}}{\partial n}\right|_{\text {porous }}-\left.v \frac{\partial u_{n}}{\partial n}\right|_{\text {fluid }}=0
\end{gathered}
$$

where $n$ is a coordinate normal to the interface with its direction from porous medium to homogenous fluid; $v_{n}$ is the velocity component perpendicular to the interface; $t$ is a coordinate perpendicular to $n$ with its direction determined by the right-handed system; $v_{t}$ is the velocity component tangential to the interface; $\beta$ is a coefficient associated with an excess viscous stress, and $\beta_{1}$ is a coefficient related to an excess inertial stress.

The Peclet number for the homogeneous fluid region is defined as

$$
P e_{f}=\frac{u_{a v_{-} f} H}{D}
$$

where $u_{a v_{-} f}$ is the average flow velocity in the homogeneous fluid region. The porous Damkohler number for cell absorption is defined as:

$$
\operatorname{Dam}_{p a}=\frac{V_{m a} \gamma_{a} h^{2}}{D_{e f f} c_{i n}}
$$

which characterizes the ratio of the time scales of substrate absorb reaction to substrate diffusion in the porous medium. The fluid Damkohler number for cell absorption is defined as:

$$
\operatorname{Dam}_{f a}=\frac{V_{m a} \gamma_{a} h}{u_{a v_{-} f} c_{i n}}
$$

which characterizes the ratio of the time scales of substrate absorb reaction to substrate convection in the fluid domain.

A lattice Boltzmann method (LBM) was applied to solve the above governing equations with the specified boundary conditions [21]. The height of the porous region $h$ was equal to that of the homogenous fluid region $H$. The length of the channel was $L=20 h$. A total number of $201 \times 81$ meshes was applied for domain discretization, which has been confirmed 
to be fine enough to obtain the grid independent results by a preliminary study. The jump parameters $\beta$ and $\beta_{1}$ was both set to be zero because the previous studies have indicated that these two parameters have relatively minor effect on the concentration distribution.

The concentration difference parameter $\kappa$ can be expressed as:

$$
\kappa=\frac{C_{\mathrm{int}}-C_{b o t}}{\operatorname{Dam}_{p a}(1-a)}=\frac{\frac{D_{e f f}\left(c_{\mathrm{int}}-c_{b o t}\right)}{h}}{V_{m a} \gamma_{a} h(1-a)}
$$

The study proposes two combined parameters which may be useful for correlating the interface concentration at various $P e_{f}, D_{p a m}$ and $K_{m}$ :

Effective distance parameter:

$$
\zeta=\frac{h}{H} \frac{X}{P e_{f}}=\frac{x}{H} \frac{1}{P e_{f}}
$$

Interface concentration reaction parameter:

$$
\xi_{k}=\frac{C_{\text {int }}-1}{\operatorname{Dam}_{p a} \lambda}
$$

where $\lambda$ is reaction rate parameter

$$
\lambda=\frac{H}{h}\left(\frac{1+K_{m}}{1+3 K_{m}}-a\right)
$$

To quantify the mass transfer resistance of the porous medium, an effectiveness factor is defined [22] as the ratio of actual reaction rate to that which would be obtained if the enzyme or cells were at the interface (that is without the porous medium diffusion resistance). In the present study, this definition is followed and the actual reaction rate is defined based on concentration flux normal to the interface. Based on the above definition, and the assumption that the flux equals the reaction, the local effectiveness factor can be expressed as:

$$
\chi=\frac{\left.\frac{d C}{d Y}\right|_{\mathrm{int}}}{\operatorname{Dam}_{p a}\left(\frac{C_{\mathrm{int}}}{C_{\mathrm{int}}+K_{m}}-a\right)}=\frac{\left.D_{\text {eff }} \frac{d c}{d y}\right|_{\mathrm{int}}}{V_{m a} \gamma_{a} h\left(\frac{c_{\mathrm{int}}}{c_{\mathrm{int}}+k_{m}}-a\right)}
$$

where $c_{\text {int }}$ is the local interface concentration.

The bioreactor efficiency is the ratio of actual reaction rate over the maximum reaction rate based on concentration at the inlet. For the present study, considering the Darcy velocity is at least one order smaller than plain fluid velocity, the convective mass transfer in porous wall may be negligible compared with convective mass transfer in plain fluid region. Thus the bioreactor efficiency may be expressed based on inlet and outlet flux difference in the plain fluid region:

$$
\eta=\frac{1-\bar{C}_{\text {out }}}{\operatorname{Dam}_{f a}\left(\frac{1}{1+K_{m}}-a\right) \frac{L}{H}}=\frac{u_{f_{-} a v} H\left(c_{\text {in }}-\bar{c}_{\text {out }}\right)}{h L\left(V_{m a} \gamma_{a} \frac{c_{\text {in }}}{c_{\text {in }}+k_{m}}-V_{m r} \gamma_{r}\right)}
$$

where $\bar{C}_{\text {out }}$ is the average outlet concentration in plain fluid region and $c_{\text {in }}$ is inlet concentration.

To evaluate the wastage of substrate in bioreactors, the utilization efficiency (or conversion rate) is defined as the ratio of actual utilized mass rate over the inlet mass rate:

$$
\eta_{u}=\frac{u_{f_{-} a v} H\left(c_{\mathrm{in}}-\bar{c}_{\mathrm{out}}\right)}{u_{f_{-} a v} H c_{\mathrm{in}}}=1-\bar{C}_{\mathrm{out}}
$$

Combining the above two equations, the utilization efficiency is expressed as:

$$
\eta_{u}=\eta \cdot \operatorname{Dam}_{f a}\left(\frac{1}{1+K_{m}}-a\right) \frac{L}{H}
$$

\section{RESUlTS AND DISCUSSION}

The concentration reaction parameter $\xi_{k}$ at different release and consumption rates is plotted as a function of effective distance parameter $\zeta=x /\left(H \cdot P e_{f}\right)$ in Fig. 2 . The results show that the concentration reaction parameter decreases with increasing effective distance parameter. The numerical results agree with the general trend of the analytical solution except the gradient is steeper. The variations of $\operatorname{Dam}_{p a}$ and $a$ do not significantly change the non-dimensional results, except for one case at large $\operatorname{Dam}_{p a}$ of 0.5 and zero release ratio $a$. The normalization of the interface concentration by $\operatorname{Dam}_{p a} \lambda$, and plotted against effective distance $x /\left(H \cdot P e_{f}\right)$, is effective in collapsing the numerical data.

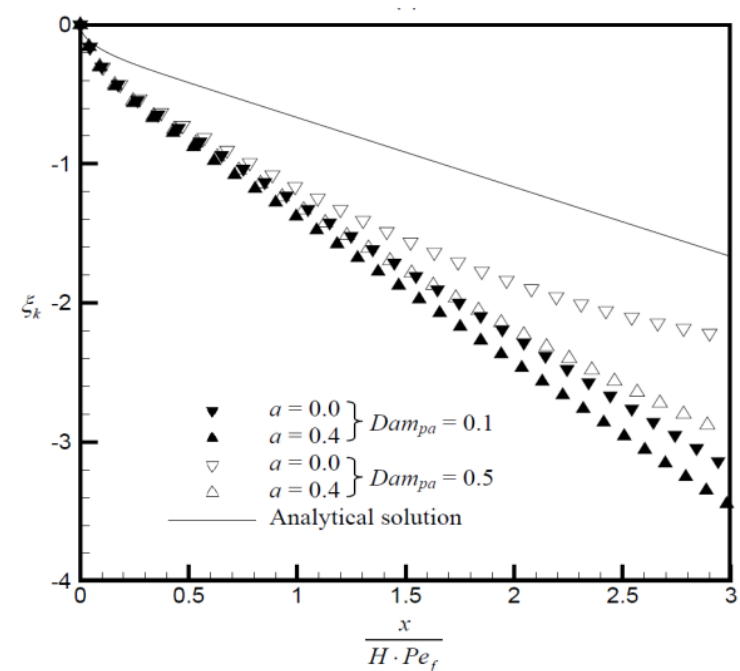

Fig. 2. Concentration reaction parameter as function of effective distance parameter when $\varepsilon=0.8$ and $K_{m}=0.128$ at different $a$ and $D_{a m}$.

The concentration difference parameter $\kappa$ at different release and consumption rates is plotted as a function of effective distance parameter $x /\left(H \cdot P e_{f}\right)$ in Fig. 3. All the data collapse together except that at large $\operatorname{Dam}_{p a}$ of 0.5 and zero release ratio $a$. The spread of data is less at smaller effective distance smaller than 2. The effects of $\operatorname{Dam}_{p a}$ and $a$ have been incorporated in the concentration difference parameter. It is noted that away from the inlet, the concentration difference parameter is around 0.5 which agrees with the analytical solution (Equation 19). 


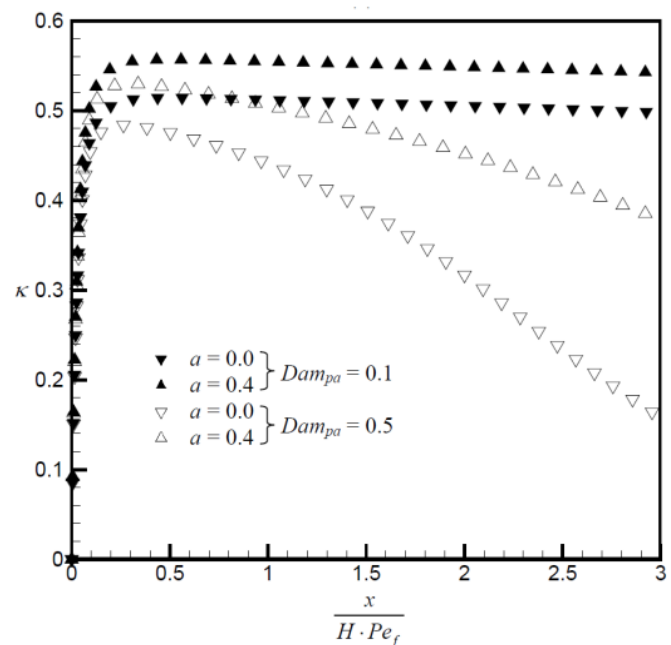

Fig. 3. Concentration difference parameter as function of effective distance parameter when $\varepsilon=0.8$ and $K_{m}=0.128$ at different $a$ and $D_{a m p a}$.

Fig. 4 presents effectiveness factor $\chi$ as a function of effective distance $\zeta$. It shows that away from the inlet, the effectiveness factor varies from around 0.9 to 0.6 . The effectiveness is lower at large $\operatorname{Dam}_{p a}$ and small release ratio $a$. With large $\operatorname{Dam}_{p a}$ (relatively large consumption), the concentration in the porous medium is much lower than that at the interface. Thus the actual reaction in the porous medium compared to that at the interface is smaller; and hence the porous medium is less effective for mass transfer across the interface. The effectiveness factor is a local parameter and it becomes lower far away from the inlet due to the lower concentration in the porous medium.

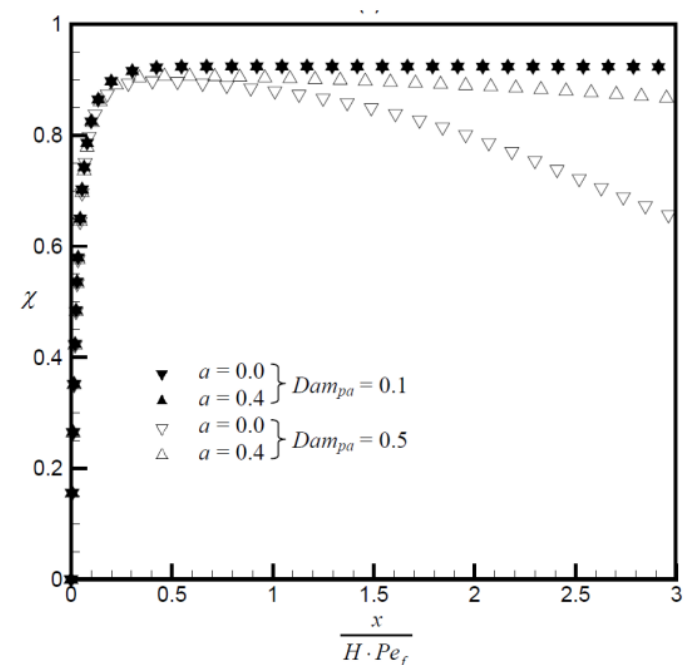

Fig. 4. Effectiveness factor as function of effective distance when $\varepsilon=0.8$ and $K_{m}=0.128$ at different $a$ and Dampa.

Fig. 5 presents the bioreactor efficiency $\eta_{u}$ as a function of effective channel length $\zeta_{L}=L /\left(H \cdot P e_{f}\right)$ at different $\operatorname{Dam}_{p a}$ and $a$. The bioreactor efficiency varies from around 0.85 to 0.6 . The efficiency is lower at larger $\operatorname{Dam}_{p a}$ and smaller $a$ due to the smaller concentration in the porous medium. At longer bioreactor length the efficiency is smaller. This is because of the decreasing interface concentration with length, which gives a lower concentration in the porous medium and hence the average reaction is low.

Fig. 6 presents utilization efficiency $\eta_{u}$ as a function of effective channel length $\zeta_{L}=L /\left(H \cdot P e_{f}\right)$ at different $\operatorname{Dam}_{p a}$ and $a$. The utilization efficiency varies from around 0 to 0.8 . The utilization efficiency is very low at small $L /\left(H \cdot P e_{f}\right)$; that is either small $L / H$ or large $P e_{f}$. This is because the convection time scale is relatively short compared to the diffusion time. Hence the substrates further away from the interface are not utilized. At larger $\operatorname{Dam}_{p a}$ the consumption is larger and hence it gives better utilization. At smaller release ratio $a$ there is a requirement for more flux and hence larger utilization. The utilization efficiency highlights the importance of effective length to achieve good utilization.

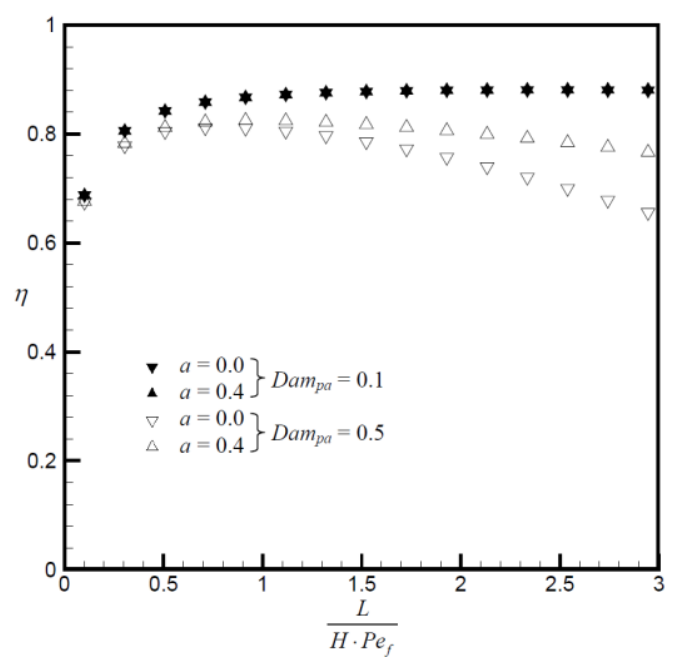

Fig. 5. Reactor efficiency as function of effective channel length when $\varepsilon=$ 0.8 and $K_{m}=0.128$ at different $a$ and Dam $_{p a}$

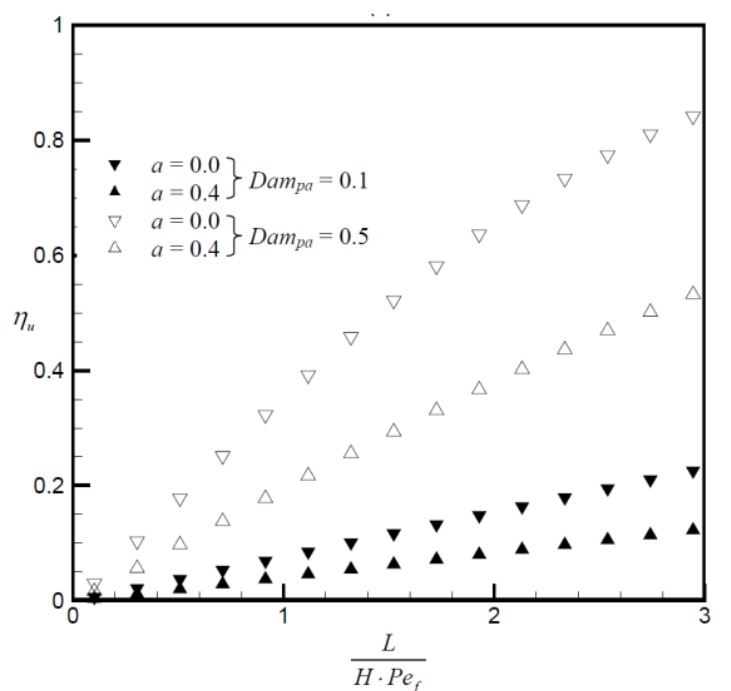

Fig. 6. Utilization efficiency as function of effective length when $\varepsilon=0.8$ and $K_{m}=0.128$ at different $a$ and Dampa.

\section{CONCLUSION}

The present work has investigated the mass transfer in a microchannel bioreactor with a porous wall in which there is a co-culture of two types of cells distributed randomly and uniformly. A group of dimensionless parameters, which can be applied to correlate the numerical data and characterize the mass transfer in the bioreactor, have been proposed based on a simplified analysis.

The velocity and concentration fields in the bioreactor have been calculated by using the LBM. It is shown that the concentration should be normalized by a parameter combining the Damkohler number Dam ${ }_{p a}$, Michaelis-Menten constant $k_{m}$ and release ratio $a$. The normalized numerical data, of the interface concentration and concentration 
difference, show satisfactory correlation when presented as a function of the effective distance $x /\left(H \cdot P e_{f}\right)$. The efficiency of the present bioreactor has been evaluated. Based on the numerical simulation, it is found that the effectiveness factor varies from around 0.9 to 0.6 , the bioreactor efficiency varies from around 0.85 to 0.6 , and the utilization efficiency varies from around 0 to 0.8 . The utilization efficiencies are very low at small $L /\left(H \cdot P e_{f}\right)$, that is either small $L / H$ or larger $P e_{f}$. This is because the convection time scale is relatively short compare to the diffusion time.

\section{REFERENCES}

[1] T. Ma, S. T. Yang, and D. A. Kniss, "Development of an in-vitro human placenta model by the cultivation of human trophoblasts in a fiber-based bioreactor system," Tissue Eng., vol. 5, pp. 91-102, 1999.

[2] F. Zhao and T. Ma, "Perfusion bioreactor system for human mesenchymal stem cell tissue engineering: Dynamic cell seeding and construct development," Biotechnol. Bioeng., vol. 91, pp. 482-493, 2005.

[3] F. Zhao, R. Chella, and T. Ma, "Effects of shear stress on 3-D human mesenchymal stem cell construct development in a perfusion bioreactor system: Experiments and hydrodynamic modelling," Biotechnol. Bioeng., vol. 96, pp. 584-595, 2007.

[4] P. Pathi, T. Ma, and B. R. Locke, "Role of nutrient supply on cell growth in bioreactor design for tissue engineering of hematopoietic cells," Biotechnol. Bioeng., vol. 89, pp. 743-758, 2005.

[5] F. Zhao, P. Pathi, W. Grayson, Q. Xing, B. R. Locke, and T. Ma "Effects of oxygen transport on 3-D human mesenchymal stem cell metabolic activity in perfusion and static cultures: Experiments and mathematical model," Biotechnol. Prog., vol. 21, pp. 1269-1280, 2005.

[6] X. B. Chen, Y. Sui, H. P. Lee, H. X. Bai, P. Yu, S. H. Winoto, and H. T. Low, "Mass transport in a microchannel bioreactor with a porous wall," J. Biomech. Eng.-T. ASME, vol. 132, pp. 061001, 2010.

[7] Y. Zeng, T. S. Lee, P. Yu, P. Roy, and H. T. Low, "Mass transport and shear stress in a microchannel bioreactor: Numerical simulation and dynamic similarity," J. Biomech. Eng.-T. ASME, vol. 128, pp. 185-193, 2006.

[8] D. C. Chow, L. A. Wenning, W. M. Miller, and E. T. Papoutsakis, "Modeling pO2 distributions in the bone marrow hematopoietic compartment. I. Krogh's model,” Biophys. J., vol. 81, pp. 675-684, 2001.

[9] R. Filion and A. S. Popel, "Intracorponary administration of fgf-2: a computational model of myocardial deposition and retention," Am. $J$. Physio. Heart Circ. Physiol., vol. 288, pp. H263-H279, 2005.

[10] Y. Zeng, T. S. Lee, P. Yu, and H. T. Low, "Numerical simulation of mass transport in a microchannel bioreactor with cell micropatterning," J. Biomech. Eng.-T., ASME, vol. 130, pp. 031018, 2008.

[11] M. Dvir-Ginzberg, I. Gamlieli-Bonshtein, R. Agbaria, and S. Cohen, "Liver tissue engineering within alginate scaffolds: effects of cell-seeding density on hepatocyte viability, morphology, and function," Tissue Eng., vol. 9, pp. 757-766, 2003.
[12] S. N. Bhatia, U. J. Balis, M. L. Yarmush, and M. Toner, "Effect of cell-cell interactions in preservation of cellular phenotype: co-cultivation of hepatocytes and nonparenchymal cells, " FASEB J., vol. 13, pp. 1883-1900, 1999.

[13] M. L. Shuler and F. Kargi, Bioprocess Engineering: Basic Concepts, Prentice Hall, 2002.

[14] Y. Zeng, T. S. Lee, P. Yu, and H. T. Low, "Numerical simulation on mass transport in a microchannel bioreactor for co-culture applications," J. Biomech. Eng.-T. ASME, vol. 129, pp. 365-373, 2007.

[15] C. T. Hsu and P. Cheng, "Thermal dispersion in a porous medium," Int. J. Heat .Mass Trans., vol. 33, pp. 1587-1597, 1990.

[16] K. Vafai and C. L. Tien, "Boundary and inertia effects on flow and heat transfer in porous media," Int. J. Heat .Mass Trans., vol. 24, pp. 195-203, 1981

[17] K. Vafai and S. J. Kim, "Fluid Mechanics of the interface region between a porous Medium and a fluid Layer - an exact solution," Int. J. Heat Fluid Flow, vol. 11, pp. 254-256, 1990.

[18] P. Yu, T. S. Lee, Y. Zeng, and H. T. Low, "A numerical method for flows in porous and homogenous fluid domains coupled at the interface by stress jump," Int. J. Numer. Methods Fluids, vol. 53, pp. 1755-1775, 2007.

[19] J. J. Valencia-López, G. Espinosa-Paredes, and J. A. Ochoa-Tapia, "Mass transfer jump condition at the boundary between a porous medium and a homogeneous fluid," J. Porous Media, vol. 6, pp. 33-49, 2003.

[20] J. A. Ochoa-Tapia, and S. Whitaker, "Momentum jump condition at the boundary between a porous medium and a homogeneous fluid: inertial effect," J Porous Media, vol. 1, pp. 201-217, 1998.

[21] H. X. Bai, P. Yu, S. H. Winoto, and H. T. Low, "Lattice Boltzmann method for flows in porous and homogenous fluid domains coupled at the interface by stress jump," International Journal for Numerical Methods in Fluids, vol. 60, pp. 691-708, 2009.

[22] A. E. Al-Muftah, and I. M. Abu-Reesh, "Effects of simultaneous internal and external mass transfer and product inhibition on immobilized enzyme-catalyzed reactor," Biochem. Eng. J., vol. 27, pp. 167-178, 2005.

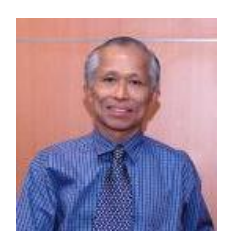

H. T. Low was born in Singapore. He graduated from the University of Sheffield, United Kingdom, in 1975 with a Bachelor of engineering (honours in mechanical engineering). $\mathrm{He}$ did his graduate studies in aerodynamics at McGill University, Montreal, Canada, and obtained a master of Engineering in 1980 and a doctor of Philosophy (Mechanical Engineering) in 1982. He had served national service in the Singapore Armed Forces. He worked as an engineer at the Public Utilities Board, Singapore, from 1977 to 1978. He is presently an associate professor at the National University of Singapore, where he has worked since 1983 . He has published about 90 papers in international refereed journals on his research areas which include computational fluid dynamics, porous flows, aerodynamics, and bioengineering. 